PROCEEDINGS OF THE

AMERICAN MATHEMATICAL SOCIETY

Volume 129, Number 10, Pages 3101-3107

S 0002-9939(01)05930-5

Article electronically published on February 15, 2001

\title{
LOCAL SOLVABILITY FOR POSITIVE COMBINATIONS OF GENERALIZED SUB-LAPLACIANS ON THE HEISENBERG GROUP
}

\author{
DETLEF MÜLLER AND ZHENQIU ZHANG
}

(Communicated by Christopher D. Sogge)

\begin{abstract}
As one step in a program to understand local solvability of complex coefficient second order differential operators on the Heisenberg group in a complete way, solvability of operators of the form $\Delta_{S, \alpha}=\Delta_{S}+i \alpha U$, where the leading term $\Delta_{S}$ is a "positive combination of generalized and degenerate generalized sub-Laplacians", has been studied in a recent article by M. Peloso, F. Ricci and the first-named author (J. Reine Angew Math. 513 (1999)). It was shown that there exists a discrete set of "critical" values $E \subset \mathbb{C}$, such that solvability holds for $\alpha \notin E$. The case $\alpha \in E$ remained open, and it is the purpose of this note to close this gap. Our results extend corresponding results in another article by the above-mentioned authors (J. Funct. Anal. 148 (1997)), by means of an even simplified approach which should allow for further generalizations.
\end{abstract}

\section{INTRODUCTION}

Let $\omega$ denote the symplectic form on $\mathbb{R}^{2 n}$ given by

$$
\omega\left(z, z^{\prime}\right):={ }^{t} z^{\prime} J z, \quad J:=\left(\begin{array}{cc}
0 & I_{n} \\
-I_{n} & 0
\end{array}\right) .
$$

The Heisenberg group $\mathbb{H}_{n}$ is $\mathbb{R}^{2 n} \times \mathbb{R}$, endowed with the group law

$$
(z, u)\left(z^{\prime}, u^{\prime}\right):=\left(z+z^{\prime}, u+u^{\prime}-\frac{1}{2} \omega\left(z, z^{\prime}\right)\right) .
$$

If we write $z=(x, y)=\left(x_{1}, \ldots, x_{n}, y_{1}, \ldots, y_{n}\right)$, a basis for the Lie algebra $\mathfrak{h}_{n}$ of $\mathbb{H}_{n}$ is given by the left-invariant vector fields

$$
\begin{aligned}
X_{j} & :=\frac{\partial}{\partial x_{j}}-\frac{1}{2} y_{j} \frac{\partial}{\partial u}, \quad j=1, \ldots, n, \\
Y_{j} & :=\frac{\partial}{\partial y_{j}}+\frac{1}{2} x_{j} \frac{\partial}{\partial u}, \quad j=1, \ldots, n, \\
U & :=\frac{\partial}{\partial u} .
\end{aligned}
$$

The non-trivial commutation relations among these vector fields are

$$
\left[X_{j}, Y_{j}\right]=U, \quad j=1, \ldots, n .
$$

Received by the editors February 3, 2000.

2000 Mathematics Subject Classification. Primary 22E30; Secondary 35A07. 
Denote by $\mathfrak{s p}(n, \mathbb{C})$ the symplectic Lie algebra, consisting of all complex $2 n \times 2 n$ matrices $S$ satisfying

$$
{ }^{t} S J+J S=0 .
$$

Let $S \in \mathfrak{s p}(n, \mathbb{C})$. Following the notation of [5] and [4], we denote by $A=\left(a_{j k}\right)$ the symmetric matrix $A:=S J$, and put

$$
\Delta_{S}:=\sum_{j, k=1}^{2 n} a_{j k} V_{j} V_{k}
$$

where $V_{j}:=X_{j}, V_{n+j}:=Y_{j}, j=1, \ldots, n$.

In [4, the situation where the matrix $S$ assumes a block diagonal form

$$
S=\left(\begin{array}{cccc}
\gamma_{1} S_{(1)} & & & \\
& \gamma_{2} S_{(2)} & & \\
& & \ddots & \\
& & & \gamma_{m} S_{(m)}
\end{array}\right)
$$

with respect to a suitable decomposition of $\mathbb{R}^{2 n}$ into symplectic subspaces has been studied, under the assumptions that $\gamma_{j} \in \mathbb{C}^{\times}=\mathbb{C} \backslash\{0\}$ and $S_{(j)}^{2}=-I, j=1, \ldots, m$. By means of Hörmander's criterion, it has been shown that for "most" of these matrices $S$, the operators $\Delta_{S}+$ lower order terms are locally non-solvable.

There are only five exceptional classes of operators of the above type to which Hörmander's criterion does not apply and which are listed in 44. In all these classes, we may assume that each of the block matrices $S_{(j)}$ is of size $2 \times 2$. Moreover, according to the classification of normal forms in [6], after applying a suitable symplectic change of coordinates, we may assume that $S_{(j)}$ is either of the form

$$
S_{(j)}=\left(\begin{array}{cc}
i \varepsilon_{j} \lambda_{j} & \lambda_{j}^{2}-1 \\
1 & -i \varepsilon_{j} \lambda_{j}
\end{array}\right), \quad \quad \text { "Type } 1 "
$$

with $\lambda_{j} \in\{-1\} \cup\left[0, \infty\left[\right.\right.$ and $\varepsilon_{j}=1$ if $\left|\lambda_{j}\right| \leq 1$, and $\varepsilon_{j}= \pm 1$ if $\lambda_{j}>1$, or of the form

$$
S_{(j)}=\left(\begin{array}{cc}
0 & i \\
i & 0
\end{array}\right) \quad \text { "Type } 3 " .
$$

Observe that the matrix $\left(\begin{array}{ll}0 & 1 \\ 1 & 0\end{array}\right)$ can be conjugated into the matrix $\left(\begin{array}{cc}1 & 0 \\ 0 & -1\end{array}\right)$ by means of the real symplectic matrix $\frac{1}{\sqrt{2}}\left(\begin{array}{cc}1 & 1 \\ -1 & 1\end{array}\right) \in \operatorname{Sp}(2, \mathbb{R})$. We may therefore assume as well that blocks of Type 3 are given by

$$
S_{(j)}=\left(\begin{array}{cc}
i & 0 \\
0 & -i
\end{array}\right),
$$

which turns out to be more convenient for our purposes.

Denote by $\sigma_{S}$ the principal symbol of $-\Delta_{S}$. If we assume that $\operatorname{Re} \sigma_{S} \geq 0$, then $\Delta_{S}$ belongs to one of the major exceptional classes listed in [4], and it follows from [4, Thm. 6.1] and (1.5) that $\Delta_{S}$ is of the form

$$
\Delta_{S}=\sum_{j=1}^{r} \gamma_{j}\left[\left(1-\lambda_{j}^{2}\right) X_{j}^{2}+Y_{j}^{2}+i \lambda_{j}\left(X_{j} Y_{j}+Y_{j} X_{j}\right)\right]+i \sum_{j=r+1}^{n} \gamma_{j}\left(X_{j} Y_{j}+Y_{j} X_{j}\right),
$$


where $0 \leq r \leq n,\left|\lambda_{j}\right| \leq 1, \gamma_{j} \in \mathbb{C}^{\times}$for $j=1, \ldots, r, \gamma_{j}>0$ for $j=r+1, \ldots, n$, and where for each $j=1, \ldots, r$ and every $\xi_{j}, \eta_{j} \in \mathbb{R}$

$$
\operatorname{Re}\left[\gamma_{j}\left[\left(1-\lambda_{j}^{2}\right) \xi_{j}^{2}+\eta_{j}^{2}+2 i \lambda_{j} \xi_{j} \eta_{j}\right]\right] \geq 0,
$$

provided we choose appropriate coordinates.

Let us assume in the sequel that $\Delta_{S}$ is of this form, and put $\Delta_{S, \alpha}:=\Delta_{S}+$ $i \alpha U, \alpha \in \mathbb{C}$. Set

$$
E^{ \pm}:=\left\{ \pm \sum_{j=1}^{r} \gamma_{j}\left(2 \ell_{j}+1\right): \ell_{1}, \ldots, \ell_{r} \in \mathbb{N}\right\}
$$

and put $E:=E^{+} \cup E^{-}$. Moreover, denote by $n_{1}, n_{2}^{+}$and $n_{2}^{-}$the number of "Type 1" blocks $S_{(j)}$ in $S$ with $\left|\lambda_{j}\right|<1, \lambda_{j}=1$ and $\lambda_{j}=-1$, respectively, and by $n_{3}$ the number of "Type 3 " blocks. We shall prove the following extension of [3] Thm. 3.2] and [4 Thms. 7.1, 7.9].

Theorem 1.1. Let $\Delta_{S}$ be as described by (1.6) and (1.7), and assume that $\operatorname{Re} \gamma_{j}>$ 0 for $j=1, \ldots, r$. Then $E$ is discrete in $\mathbb{C}$, and the following holds:

(i) If $\alpha \notin E$, then $\Delta_{S, \alpha}$ is locally solvable.

(ii) If $\alpha \in E^{-}$, then $\Delta_{S, \alpha}$ is locally solvable if and only if $n_{2}^{+}+n_{3}>0$.

(iii) If $\alpha \in E^{+}$, then $\Delta_{S, \alpha}$ is locally solvable if and only if $n_{2}^{-}+n_{3}>0$.

Remark 1.2. (1.7) implies that $\operatorname{Re} \gamma_{j} \geq 0$ for $j=1, \ldots, r$. Moreover, if $\operatorname{Re} \gamma_{j}=0$, then $\lambda_{j}=0$, again by (1.7). Therefore, if $\operatorname{Re} \gamma_{j}=0$ for every $j$, then $\Delta_{S}$ is a multiple of a real-coefficient operator, and local solvability of $\Delta_{S, \alpha}$ can in this case be described by means of [5]. Notice that in this case the exceptional set may be non-discrete. We do not know at present what will happen if $\operatorname{Re} \gamma_{j}=0$ only for some $j \in\{1, \ldots, r\}$.

\section{Proof of Theorem 1.1}

The negative results are proved in the same way as Theorem 7.1 in [4]. For instance, if $\alpha \in E^{+}$and $n_{2}^{-}=n_{3}=0$, then

$$
\Delta_{S}=\sum_{j=1}^{n} \gamma_{j} \Delta_{S_{(j)}}=\sum_{j=1}^{n} \gamma_{j}\left[\left(1-\lambda_{j}^{2}\right) X_{j}^{2}+Y_{j}^{2}+i \lambda_{j}\left(X_{j} Y_{j}+Y_{j} X_{j}\right)\right]
$$

where $\left|\lambda_{j}\right|<1$ or $\lambda_{j}=1$. Let $\pi_{\mu}$ denote the Schrödinger representation of $\mathbb{H}_{n}$ with parameter $\mu \in \mathbb{R}^{\times}$, acting on the Hilbert space $L^{2}\left(\mathbb{R}^{n}\right)$.

If $\left|\lambda_{j}\right|<1$, then there exist Schwartz functions $\varphi_{\ell_{j}}^{\mu}=\varphi_{\ell_{j}}^{\mu}\left(t_{j}\right)$ on $\mathbb{R}$ such that

$$
d \pi_{\mu}\left(\Delta_{S_{(j)}}\right) \varphi_{\ell_{j}}^{\mu}=-|\mu|\left(2 \ell_{j}+1\right) \varphi_{\ell_{j}}^{\mu}
$$

for every $\ell_{j} \in \mathbb{N}$ (compare [4, Prop. 7.2]).

And, if $\lambda_{j}=1$, then

$$
d \pi_{\mu}\left(\Delta_{S_{(j)}}-i U\right)=-\mu t_{j}\left(2 \frac{\partial}{\partial t_{j}}+\mu t_{j}\right)
$$

hence

$$
d \pi_{\mu}\left(\Delta_{S_{(j)}}-i U\right) e^{-\mu t_{j}^{2} / 4}=0 .
$$

Choosing $\mu>0$ and putting $\varphi_{0}^{\mu}\left(t_{j}\right):=e^{-\mu t_{j}^{2} / 4}$, we see that $\varphi_{0}^{\mu} \in \mathcal{S}(\mathbb{R})$ and

$$
d \pi_{\mu}\left(\Delta_{S_{(j)}}-i U\right) \varphi_{0}^{\mu}=0 .
$$


More generally, following [3], define operators $A:=-\mu t_{j}, B:=2 \frac{\partial}{\partial t_{j}}+\mu t_{j}$, and put $\varphi_{\ell_{j}}^{\mu}:=A^{\ell_{j}} \varphi_{0}^{\mu}$. Since $d \pi_{\mu}\left(\Delta_{S_{(j)}}-i U\right)=A B$ and $[B, A]=-2 \mu$, by induction over $\ell_{j} \in \mathbb{N}$ one obtains

$$
d \pi_{\mu}\left(\Delta_{S_{(j)}}-i U\right) \varphi_{\ell_{j}}^{\mu}=-\mu 2 \ell_{j} \varphi_{\ell_{j}}^{\mu}
$$

hence

$$
d \pi_{\mu}\left(\Delta_{S_{(j)}}\right) \varphi_{\ell_{j}}^{\mu}=-|\mu|\left(2 \ell_{j}+1\right) \varphi_{\ell_{j}}^{\mu},
$$

where $\varphi_{\ell_{j}}^{\mu} \in \mathcal{S}(\mathbb{R})$. Consequently, if we define $\varphi_{\ell}^{\mu}\left(t_{1}, \ldots, t_{n}\right):=\varphi_{\ell_{1}}^{\mu}\left(t_{1}\right) \ldots \varphi_{\ell_{n}}^{\mu}\left(t_{n}\right)$, for $\ell=\left(\ell_{1}, \ldots, \ell_{n}\right) \in \mathbb{N}^{n}$, then $\varphi_{\ell}^{\mu} \in \mathcal{S}\left(\mathbb{R}^{n}\right)$, and

$$
d \pi_{\mu}\left({ }^{t} \Delta_{S, \alpha}\right) \varphi_{\ell}^{\mu}=-|\mu|\left(\sum_{j=1}^{n} \gamma_{j}\left(2 \ell_{j}+1\right)-\alpha\right) \varphi_{\ell}^{\mu},
$$

provided $\mu>0$. This shows that $d \pi_{\mu}\left({ }^{t} \Delta_{S, \alpha}\right)$ annihilates a non-trivial Schwartz function; hence $\Delta_{S, \alpha}$ is locally non-solvable.

Consider next the case $\alpha \notin E$. Put

$$
A:=\sum_{j} \operatorname{Re} \gamma_{j}, \quad a:=\min _{j} \operatorname{Re} \gamma_{j}
$$

and define

$$
\Gamma_{t, S}^{\mu}(z):=\frac{|\mu|^{n}}{(4 \pi)^{n} \prod_{j=1}^{n} \sinh \left(\gamma_{j} t\right)} e^{-\frac{|\mu|}{4}\left(\sum_{j=1}^{n} \operatorname{coth}\left(\gamma_{j} t\right)^{t} z_{j} J_{1} S_{(j)} z_{j}\right)}
$$

for $z=\left(z_{1}, \ldots, z_{n}\right) \in \mathbb{R}^{2 n}$, where $z_{j}=\left(x_{j}, y_{j}\right)$ and $J_{1}=\left(\begin{array}{rr}0 & 1 \\ -1 & 0\end{array}\right)$. It follows from the arguments in [4, Section 7] that for $\alpha \in \mathbb{C}$ with $|\operatorname{Re} \alpha|<A$, a tempered distribution $F_{\alpha}$ is defined on $\mathbb{H}_{n}$ by means of the formula

$$
\left\langle F_{\alpha}, \varphi\right\rangle:=-\frac{1}{2 \pi} \int_{0}^{\infty} \int_{\mathbb{R}^{\times}} e^{-\alpha(\operatorname{sgn} \mu) t}\left\langle\Gamma_{t, S}^{\mu}, \varphi^{-\mu}\right\rangle \frac{i \mu}{|\mu|} d \mu d t, \quad \varphi \in \mathcal{S}\left(\mathbb{H}_{n}\right),
$$

which satisfies

$$
\Delta_{S, \alpha} F_{\alpha}=U \delta_{0} .
$$

Here, $\langle$,$\rangle denotes the pairing between \mathcal{S}^{\prime}$ and $\mathcal{S}$, and $\varphi^{\mu}$ the partial Fourier transform

$$
\varphi^{\mu}(z):=\int_{\mathbb{R}} \varphi(z, u) e^{-i \mu u} d u, \mu \in \mathbb{R}
$$

of $\varphi$ along the center.

Moreover, the family of distributions $\left\{F_{\alpha}\right\}_{|\operatorname{Re} \alpha|<A}$ is analytic.

As for values of $\alpha$ with $|\operatorname{Re} \alpha|>A$, fix $m \in \mathbb{N}$ and put $G_{\alpha}=G_{\alpha, m}:=U^{m} F_{\alpha}$, first for $|\operatorname{Re} \alpha|<A$, i.e.

$$
\left\langle G_{\alpha}, \varphi\right\rangle=-\frac{1}{2 \pi} \int_{0}^{\infty} \int_{\mathbb{R}^{\times}} e^{-\alpha(\operatorname{sgn} \mu) t}\left\langle\Gamma_{t, S}^{\mu}, \varphi^{-\mu}\right\rangle \frac{(i \mu)^{m+1}}{|\mu|} d \mu d t .
$$

The arguments in [4, Section 7] can easily be adapted to prove the following:

Proposition 2.1. The family of distributions $G_{\alpha}$ admits an analytic extension to a family of distributions $\left\{G_{\alpha}\right\}_{\alpha \in D_{m} \backslash E}$, where $D_{m}$ denotes the domain $D_{m}:=\{\alpha \in$ $\mathbb{C}:|\operatorname{Re} \alpha|<A+2 m a\}$. Moreover,

$$
\Delta_{S, \alpha} G_{\alpha}=U^{m} \delta_{0}
$$


This result implies in particular that $\Delta_{S, \alpha}$ is locally solvable for $\alpha \notin E$.

There remains the case where $\alpha \in E^{ \pm}$. Then choose $m \in \mathbb{N}$ sufficiently large, so that $\alpha \in D_{m}$. For $\beta \in D_{m} \backslash E$ sufficiently close to $\alpha$, we have

$$
\Delta_{S, \alpha} G_{\beta}=U^{m+1} \delta_{0}+i(\alpha-\beta) U G_{\beta} .
$$

The crucial point in proving local solvability for $\Delta_{S, \alpha}$ is the following:

Lemma 2.2. If $\alpha \in E^{ \pm}$and $n_{2}^{\mp}+n_{3}>0$, then there exists a right-invariant locally solvable differential operator $D^{(r)}$ on $\mathbb{H}_{n}$, such that the family of distributions $\left\{D^{(r)} G_{\beta}\right\}_{\beta \in D_{m} \backslash E}$ extends analytically into the point $\alpha$.

In fact, given this lemma, we may adapt the proof of [3. Lemma 2.2] as follows. Define the distribution $K_{\alpha}$ by $\left\langle K_{\alpha}, \varphi\right\rangle:=\lim _{\beta \rightarrow \alpha}\left\langle D^{(r)} G_{\beta}, \varphi\right\rangle$. Since, according to (2.6),

$$
\Delta_{S, \alpha}\left(D^{(r)} G_{\beta}\right)=D^{(r)}\left(\Delta_{S, \alpha} G_{\beta}\right)=U^{m+1} D^{(r)} \delta_{0}+i(\alpha-\beta) U\left(D^{(r)} G_{\beta}\right),
$$

we obtain in the limit as $\beta \in D_{m} \backslash E$ tends to $\alpha$ (taken in the distributional sense) that

$$
\Delta_{S, \alpha} K_{\alpha}=U^{m+1} D^{(r)} \delta_{0}
$$

Since $U^{m+1}$ and $D^{(r)}$ are locally solvable, this implies local solvability of $\Delta_{S, \alpha}$.

There remains to prove Lemma 2.2. To this end, let us first consider $G_{\beta}$ for $|\operatorname{Re} \beta|<A$, so that $G_{\beta}$ is given by

$$
\left\langle G_{\beta}, \varphi\right\rangle=-\frac{1}{2 \pi} \int_{0}^{\infty} \int_{\mathbb{R}^{\times}} e^{-\beta(\operatorname{sgn} \mu) t}\left\langle\Gamma_{t, S}^{\mu}, \varphi^{-\mu}\right\rangle \frac{(i \mu)^{m+1}}{|\mu|} d \mu d t .
$$

Moreover, if we put $\check{g}(z):=g(-z)$, then

$$
\left\langle\Gamma_{t, S}^{\mu}, \varphi^{-\mu}\right\rangle=\left\langle\widehat{\Gamma_{t, S}^{\mu}}, \widehat{\breve{\varphi}^{-\mu}}\right\rangle
$$

where by 4$] \widehat{\Gamma_{t, S}^{\mu}}$ is given by

$$
\widehat{\Gamma_{t, S}^{\mu}}(\zeta)=\frac{1}{\sigma(t)} e^{-\frac{1}{|\mu|} q_{t}(\zeta)},
$$

with

$$
\begin{aligned}
\sigma(t) & :=\prod_{j=1}^{n} \cosh \left(\gamma_{j} t\right) \\
q_{t}(\zeta) & :=\sum_{j=1}^{r} \tanh \left(\gamma_{j} t\right)\left[\left(1-\lambda_{j}^{2}\right) \xi_{j}^{2}+\eta_{j}^{2}+2 i \lambda_{j} \xi_{j} \eta_{j}\right]+\sum_{j=r+1}^{n} \tanh \left(\gamma_{j} t\right) 2 i \xi_{j} \eta_{j},
\end{aligned}
$$

if $\zeta_{j}=\left(\xi_{j}, \eta_{j}\right)$.

Consider the right-invariant real vector fields

$$
X_{j}^{(r)}:=\frac{\partial}{\partial x_{j}}+\frac{1}{2} y_{j} \frac{\partial}{\partial u}, Y_{j}^{(r)}:=\frac{\partial}{\partial y_{j}}-\frac{1}{2} x_{j} \frac{\partial}{\partial u},
$$

and put

$$
q_{j}\left(\xi_{j}, \eta_{j}\right):= \begin{cases}\left(1-\lambda_{j}^{2}\right) \xi_{j}^{2}+\eta_{j}^{2}+2 i \lambda_{j} \xi_{j} \eta_{j} & \text { if } j=1, \ldots, r, \\ 2 i \xi_{j} \eta_{j} & \text { if } j=r+1, \ldots, n .\end{cases}
$$


For $k \in \mathbb{N}$, by $(2.8)$ we have

$$
\begin{aligned}
\left\langle\Gamma_{t, S}^{\mu},\left(\left(-X_{j}^{(r)}\right)^{k} \varphi\right)^{-\mu}\right\rangle & =\left\langle\left(\frac{\partial}{\partial x_{j}}+i \frac{\mu}{2} y_{j}\right)^{k} \Gamma_{t, S}^{\mu}, \varphi^{-\mu}\right\rangle \\
& =\left\langle\left(-\frac{\mu}{2} \frac{\partial}{\partial \eta_{j}}+i \xi_{j}\right)^{k} \widehat{\Gamma_{t, S}^{\mu}}, \widehat{\varphi^{-\mu}}\right\rangle .
\end{aligned}
$$

And, if $j \geq r+1$, then $-\frac{\mu}{2} \frac{\partial}{\partial \eta_{j}} q_{j}=-i \mu \xi_{j}$, so that

$$
\left(-\frac{\mu}{2} \frac{\partial}{\partial \eta_{j}}+i \xi_{j}\right)^{k} \widehat{\Gamma_{t, S}^{\mu}}=\left(i \xi_{j}\right)^{k}\left(1+(\operatorname{sgn} \mu) \tanh \left(\gamma_{j} t\right)\right)^{k} \widehat{\Gamma_{t, S}^{\mu}} .
$$

Similarly,

$$
\left\langle\Gamma_{t, S}^{\mu},\left(\left(-Y_{j}^{(r)}\right)^{k} \varphi\right)^{-\mu}\right\rangle=\left\langle\left(\frac{\mu}{2} \frac{\partial}{\partial \xi_{j}}+i \eta_{j}\right)^{k} \widehat{\Gamma_{t, S}^{\mu}}, \widehat{\breve{\varphi}^{-\mu}}\right\rangle,
$$

and $\frac{\mu}{2} \frac{\partial}{\partial \xi_{j}} q_{j}=i \mu \lambda_{j} \eta_{j}$, if $j \leq r$ and $\left|\lambda_{j}\right|=1, \frac{\mu}{2} \frac{\partial}{\partial \xi_{j}} q_{j}=i \mu \eta_{j}$, if $j \geq r+1$, so that

$$
\begin{aligned}
& \left(\frac{\mu}{2} \frac{\partial}{\partial \xi_{j}}+i \eta_{j}\right)^{k} \widehat{\Gamma_{t, S}^{\mu}} \\
& \quad= \begin{cases}\left(i \eta_{j}\right)^{k}\left(1-\lambda_{j}(\operatorname{sgn} \mu) \tanh \left(\gamma_{j} t\right)\right)^{k} \widehat{\Gamma_{t, S}^{\mu}} & \text { if } j \leq r,\left|\lambda_{j}\right|=1, \\
\left(i \eta_{j}\right)^{k}\left(1-(\operatorname{sgn} \mu) \tanh \left(\gamma_{j} t\right)\right)^{k} \widehat{\Gamma_{t, S}^{\mu}} & \text { if } j \geq r+1 .\end{cases}
\end{aligned}
$$

Now assume first that $n_{3}>0$, so that $r<n$. By $(2.10)$, we have

$$
\left|e^{-\beta(\operatorname{sgn} \mu) t} \widehat{\Gamma_{t, S}^{\mu}}(\zeta)\right| \leq 2^{n} e^{-(\operatorname{Re} \beta(\operatorname{sgn} \mu)+A) t},
$$

so that by (2.11),

$$
\begin{aligned}
& \left|e^{-\beta(\operatorname{sgn} \mu) t}\left\langle\Gamma_{t, S}^{\mu},\left(\left(-X_{n}^{(r)}\right)^{k} \varphi\right)^{-\mu}\right\rangle\right| \\
& \quad \leq \begin{cases}e^{(-\operatorname{Re} \beta-A) t}\left\|\varphi^{-\mu}\right\|_{\mathcal{S}} & \text { if } \mu>0, \\
e^{\left(\operatorname{Re} \beta-A-2 k \gamma_{n}\right) t}\left\|\varphi^{-\mu}\right\|_{\mathcal{S}} & \text { if } \mu<0\end{cases}
\end{aligned}
$$

where $\|\cdot\|_{\mathcal{S}}$ denotes a suitable continuous norm on $\mathcal{S}\left(\mathbb{R}^{2 n}\right)$. Since $\left\langle\left(X_{n}^{(r)}\right)^{k} G_{\beta}, \varphi\right\rangle=$ $\left\langle G_{\beta},\left(-X_{n}^{(r)}\right)^{k} \varphi\right\rangle$, this shows that the family of distributions $D^{(r)} G_{\beta}$, with $D^{(r)}:=$ $\left(X_{n}^{(r)}\right)^{k}$, extends analytically to the region $-A<\operatorname{Re} \beta<A+2 k \gamma_{n}$.

Similarly, from $(2.12)$ we find that $D^{(r)} G_{\beta}$ extends analytically to the region $-A-2 k \gamma_{n}<\operatorname{Re} \beta<A$, if we choose $D^{(r)}:=\left(Y_{n}^{(r)}\right)^{k}$. This proves Lemma 2.2 for the case $n_{3}>0$.

Assume finally that $n_{3}=0$, and that $\alpha \in E^{+}$and $n_{2}^{-}>0$ (the case $\alpha \in E^{-}$and $n_{2}^{+}>0$ can be treated in an analogous way). We may then assume that $\lambda_{n}=-1$.

Choosing $D^{(r)}:=\left(Y_{n}^{(r)}\right)^{k}$ and arguing as before, we deduce from (2.12) that we get the analogue to estimate (2.13), only with $X_{n}^{(r)}$ replaced by $Y_{n}^{(r)}$. Thus, the family of distributions $D^{(r)} G_{\beta}$ extends analytically to the region $-A<\operatorname{Re} \beta<$ $A+2 k \operatorname{Re} \gamma_{n}$, which contains the point $\alpha$, if $k$ is sufficiently large.

This concludes the proof of Lemma 2.2, and hence of Theorem 1.1.

\section{REFERENCES}

[1] L. Corwin, L.P. Rothschild, Necessary conditions for local solvability of homogeneous left invariant differential operators on nilpotent Lie groups, Acta Math. 147 (1981), 265-288. MR 83b:22010 
[2] F. De Mari, M. M. Peloso, F. Ricci, Analysis of second order differential operators with complex coefficients on the Heisenberg group, J. Reine Angew. Math. 464 (1995), 67-96. MR 96f:22011

[3] D. Müller, M. M. Peloso, F. Ricci, On the solvability of homogeneous left-invariant differential operators on the Heisenberg group, J. Funct. Anal. 148 (1997), 368-383. MR 98k:35024

[4] D. Müller, M. M. Peloso, F. Ricci, On local solvablity for complex coefficient differential operators on the Heisenberg group, J. Reine Angew. Math. 513 (1999), 181-234. MR 2000h:35003

[5] D. Müller, F. Ricci, Analysis of second order differential operators on the Heisenberg group II, J. Funct. Anal. 108 (1992), 296-346. MR 93j:22019

[6] D. Müller, C. Thiele, Normal forms for involutive complex Hamiltonian matrices under the real symplectic group. J. Reine Angew. Math. 513 (1999), 97-114. MR 2000h:15017

Mathematisches Seminar, C. A. - Universität Kiel, Ludewig-Meyn-Str. 4, D-24098 Kiel, Germany

E-mail address: mueller@math.uni-kiel.de

Department of Mathematics, Tianjin University 300072, Tianjin, People's Republic OF CHINA

E-mail address: zqzhangmath@yahoo.com 